\title{
Polariton Condensation in Dynamic Acoustic Lattices
}

\author{
E. A. Cerda-Méndez, ${ }^{1}$ D. N. Krizhanovskii, ${ }^{2}$ M. Wouters, ${ }^{3}$ R. Bradley,${ }^{2}$ K. Biermann, ${ }^{1}$ K. Guda, ${ }^{2}$ R. Hey, ${ }^{1}$ P. V. Santos, ${ }^{1}$ \\ D. Sarkar, ${ }^{2}$ and M. S. Skolnick ${ }^{2}$ \\ ${ }^{1}$ Paul-Drude-Institut für Festkörperelektronik, Berlin, Germany \\ ${ }^{2}$ Department of Physics and Astronomy, University of Sheffield, Sheffield S3 7RH, United Kingdom \\ ${ }^{3}$ ITP, Ecole Polytechnique Fédérale de Lausanne, 1015 Lausanne, Switzerland
}

(Received 7 June 2010; revised manuscript received 6 August 2010; published 9 September 2010)

\begin{abstract}
We demonstrate that the tunable potential introduced by a surface acoustic wave on a homogeneous polariton condensate leads to fragmentation of the condensate into an array of wires which move with the acoustic velocity. Reduction of the spatial coherence of the condensate emission along the surface acoustic wave direction is attributed to the suppression of coupling between the spatially modulated condensates. Interparticle interactions observed at high polariton densities screen the acoustic potential, partially reversing its effect on spatial coherence.
\end{abstract}

DOI: 10.1103/PhysRevLett.105.116402

Microcavity polaritons are hybrid light-matter quasiparticles arising from the strong coupling between excitons and photons confined in semiconductor microcavities. Their photonic character lends them very low masses (on the order of $10^{-5}$ of the electron mass), which favors condensation at low injected polariton densities [1]. The properties of this solid-state system can be controlled by structure design and manipulated and probed by external light beams. Unlike better known Bose-Einstein condensates (BECs) [2] such as cold atoms or liquid helium, polariton condensates are intrinsically out-of-equilibrium [3]. They exhibit characteristic condensate properties such as vorticity [4], extended spatial [1], and temporal coherence [5] as well as collective fluid behavior [6].

Cold atoms and atomic BECs in optical lattices have attracted significant interest, providing access to the coherent transport properties associated with matter field coherence or decoherence [7]. In contrast, investigation of a spatially modulated polariton condensate, a nonequilibrium and strongly interacting light-matter system, provides a highly complementary insight into this field. Polariton condensates in a static lateral periodic potential have been addressed [8]. However, polariton condensation in a strong tunable periodic potential, which is essential to study coherence or decoherence of this system, remains unexplored. Recently localization or delocalization of noncondensed excitons in coupled quantum wells was reported [9].

In this Letter, we achieve polariton condensation in $\mu \mathrm{m}$-sized periodic tunable potentials created by coherent surface acoustic waves (SAWs). We show that the application of the SAW to a uniform extended condensate creates an array of condensate wires trapped at the minima of the SAW potential, which moves with the acoustic velocity. The observed reduction of the spatial coherence of the array along the SAW propagation direction with increasing acoustic intensity is attributed to the suppressed coupling between the wires, which leads to independent
PACS numbers: 71.36.+c, 42.50.-p, 63.20.kk, 73.21.Cd

phases between adjacent condensates. We also observe coherence reduction along the SAW wires, which we suggest arises from increased phase fluctuations as the dimensionality is reduced. Finally, nonlinear interparticle interactions at high quasiparticle densities screen the applied acoustic potential, thus partially reversing the effect of the SAW.

We study GaAs-based microcavities with six $15 \mathrm{~nm}$ thick quantum wells grown by molecular beam epitaxy. The microcavities have a Rabi splitting of $6 \mathrm{meV}$ and a quality factor $Q \sim 2000$ with nearly zero detuning between the cavity and the exciton modes at $k=0$. The condensates were generated at $5 \mathrm{~K}$ by pumping with a continuous wave laser with in-plane wave vector $\mathbf{k}^{(\mathbf{p})}=\left[k_{x}^{(p)}, k_{y}^{(p)}\right]=$ $(1.8,0) \mu \mathrm{m}^{-1}$ [Fig. 1(a)] in resonance with the lower polariton (LP) branch. In this optical parametric oscillator (OPO) configuration [Fig. 1(a)] [6,10], two pump polaritons scatter into states fulfilling energy and momentum conservation. As a result, three coupled macroscopically occupied states (pump, signal, and idler) are formed. The phase of the signal is independent of that of the pump laser [11], implying the spontaneous breakdown of the U(1) symmetry at the phase transition as in a standard BEC [12].

The spatial modulation of the polariton potential by acoustic fields was introduced by a SAW of wavelength $\lambda_{\text {SAW }}=8 \mu \mathrm{m}$ propagating along a nonpiezoelectric $\langle 100\rangle$ surface direction [the $y$ direction in Fig. 1(b)]. The use of this type of SAW, which does not carry a longitudinal piezoelectric field, is essential to prevent field-induced exciton ionization [13] and to observe condensation. The SAWs were generated by interdigital transducers placed on a piezoelectric $\mathrm{ZnO}$ island on the sample surface [Fig. 1(b)] [13]. Below we state the amplitude $u$ of the acoustic field in terms of square root of the nominal $\mathrm{rf}$ power $\left(u \sim \sqrt{P_{\mathrm{rf}}}\right)$ applied to the interdigital transducer.

The SAW induces a spatial modulation of the polariton energy along the $y$ direction [cf. Figure 1(b)] through two mechanisms: (i) a type I deformation potential modulation 


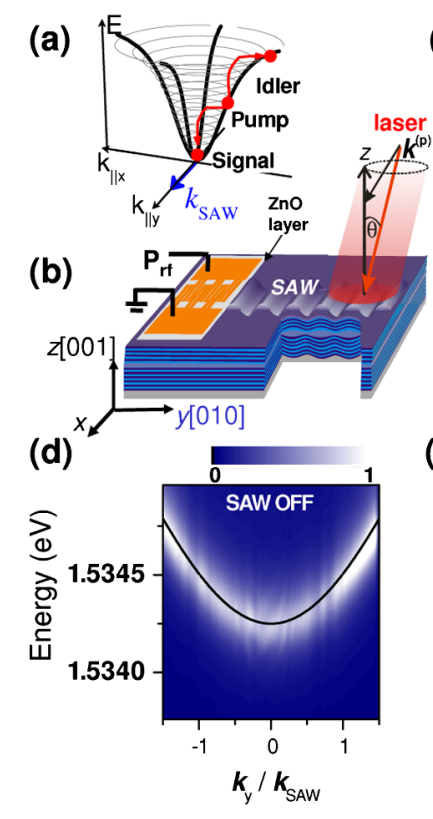

(c)

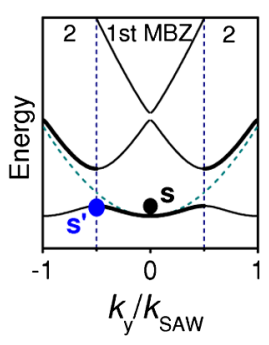

(e)

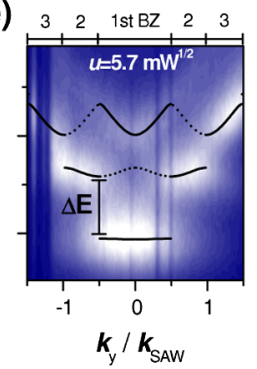

FIG. 1 (color online). (a) Schematic diagram of a polariton optical parametric oscillator. (b) Sample structure with SAWs propagating along the $y=[010]$ direction of the (001) microcavity surface. The polaritons are excited by a laser beam incident in the $(x, z)$ plane. (c) Folded dispersion of the lower polariton (LP) (d), (e) LP dispersion recorded under weak optical excitation $\left(I_{p}=20.8 \mathrm{~W} / \mathrm{cm}^{2}\right)$ in the absence and presence of a SAW $\left(u=5.65 \mathrm{~mW}^{1 / 2}\right)$, respectively. The lines display the calculated dispersion (the dotted lines indicate optically inactive states).

of the quantum wells band gap [13] and (ii) an in-phase modulation of the microcavity resonance energy [14]. This spatial and temporal modulation creates a one-dimensional (1D) lattice for polaritons, where the energy $(E)$ vs in-plane wave vector $\left[\mathbf{k}=\left(k_{x}, k_{y}\right)\right]$ dispersion is folded along $k_{y}$ within a mini-Brillouin zone (MBZ) of dimension $k_{\mathrm{SAW}}=$ $2 \pi / \lambda_{\mathrm{SAW}}$. Figure 1(c) compares the unperturbed polariton dispersion (i.e., calculated without acoustic excitation, dashed lines) with the corresponding dispersion with a SAW displayed in the extended (thick solid line) and reduced (thin line) zone schemes [15]. The anticrossing of the folded branches induces energy gaps in the center and at the edges of the MBZ. For optical excitation densities $I_{p}$ below the $\mathrm{OPO}$ condensation threshold $I_{\mathrm{th}}^{\mathrm{OPO}}=$ $600 \mathrm{~W} / \mathrm{cm}^{2}$, the far-field photoluminescence (PL) reveals the one-particle polariton dispersion, as illustrated in Figs. 1(d) and 1(e) in the absence and presence of a SAW, respectively. The well-resolved energy gaps in the center and at the edges of the first and second MBZs (i.e., at $n k_{\mathrm{SAW}} / 2= \pm n \pi / \lambda_{\mathrm{SAW}}$, where $n$ is an integer) indicate the formation of a polariton lattice [14]. The superimposed lines display the dispersion calculated using a perturbative approach [14]. For the small acoustic modulation amplitudes used in the present studies (up to $0.36 \mathrm{meV}$ ), the width $\Delta E$ of the gap at the edges of the first MBZ [see Fig. 1(e)] becomes essentially equal to the peak-to-peak amplitude modulation $V_{\mathrm{SAW}}\left(V_{\mathrm{SAW}} \sim u\right)$ of the LP branch [15].

Above the OPO threshold, self-organization sets in [16], forming a signal condensate at $\mathbf{k} \sim 0$ containing $\sim 10^{3}$ particles as well as a weaker idler at $\mathbf{k} \sim \mathbf{2} \mathbf{k}^{(\mathbf{p})}$. Figures 2(a)2(c) display $E$ vs $k_{y}$ images of the signal emission recorded under increasing SAW powers for a fixed optical excitation power density of $I_{p}=2 I_{\mathrm{th}}^{\mathrm{OPO}}=1.2 \times 10^{3} \mathrm{~W} / \mathrm{cm}^{2}$ distributed over a $50 \mu \mathrm{m}$ diameter Gaussian spot. In the absence of a SAW [Fig. 2(a)], a condensate is formed at $k=0$ and energy of $1.5345 \mathrm{eV}$, which is approximately $0.4 \mathrm{meV}$ above the bottom of the LP branch below threshold. The blueshift arises from interparticle interactions between polaritons in the OPO modes [17].

When a SAW is applied the signal emission broadens in momentum space [Figs. 2(b) and 2(c)]. We note that the linewidth of the total stimulated polariton emission (resolution limited to a FWHM of $\sim 60 \mu \mathrm{eV}$, which is one third of the subthreshold value), remains constant and much smaller than the SAW modulation amplitude over the whole range of acoustic powers, thus indicating persistence of temporal coherence. In addition, the condensate emission intensity does not change with SAW power, indicating negligible effect of the SAW on the pump rate and losses in the system.

As will be further justified below, the SAW potential fragments the original extended condensate into an array of identical, weakly interacting condensates confined at the minima of the acoustic potential, thus forming the condensate wires oriented along the SAW wave fronts illustrated
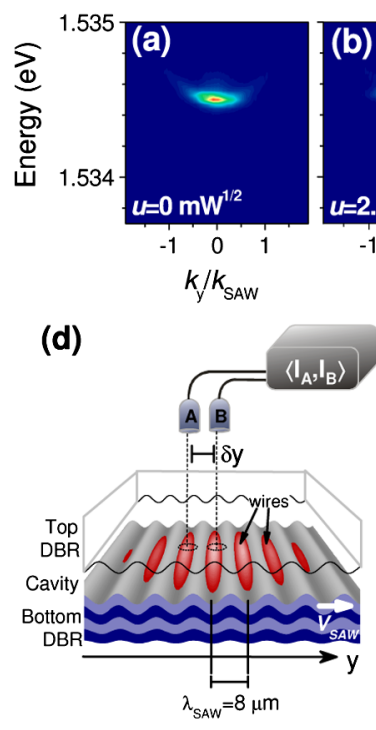
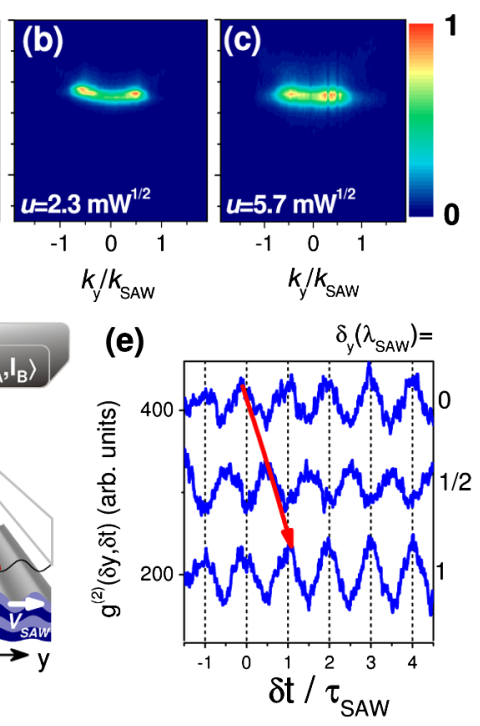

FIG. 2 (color online). (a),(c) Energy vs $k_{y}$ images of condensates for different SAW amplitudes $(u)$ at fixed pump power $I_{p}=$ $2 I_{\text {th }}^{\mathrm{OPO}}=1.2 \times 10^{3} \mathrm{~W} / \mathrm{cm}^{2}$. (d) Correlation setup to measure the $g^{(2)}(\delta y, \delta \tau)$ function of moving condensates using two detectors (A and B) separated by a distance $\delta y$. (e) $g^{(2)}(\delta y, \delta \tau)$ histograms measured at SAW amplitude $u=3.5 \mathrm{~mW}^{1 / 2}$ at different $\delta y=$ $m \lambda_{\text {SAW }} / 2(m=0,0.5$, and 1$)$. The curves are shifted down along the vertical axis for clarity. 
in Fig. 2(d). Each condensate wire moves with the acoustic velocity $v_{\mathrm{SAW}}=3 \times 10^{3} \mathrm{~m} / \mathrm{s}$ and emits independently from the others at high acoustic powers. The flattening of the lowest polariton branch [Fig. 1(e)] and the $k_{y}$ spreading of the emission [Figs. 2(b) and 2(c)] are attributed to the small width $\Delta y \leq \lambda_{\mathrm{SAW}} / 2$ of the wires in accordance with Heisenberg's uncertainty principle $\left(\Delta k_{y} \geq \pi / \Delta y \sim\right.$ $\left.2 \pi / \lambda_{\mathrm{SAW}}\right)$. Their spectral emission energies, however, overlap within the measured linewidths, thus attesting to the high homogeneity of the modulation potential.

Interestingly, at intermediate SAW amplitudes $[u=$ $2.3 \mathrm{~mW}^{1 / 2}$, cf. Fig. 2(b)] the emission peaks not around $k_{y}=0$ but at $k_{y}= \pm k_{\mathrm{SAW}} / 2$, corresponding to the $s^{\prime}$ states in Fig. 1(c) at the edge of the lowest folded branch. A likely explanation for the $s^{\prime}$-state emission arises from the spatial modulation of the pump polariton density by SAW. The excitation of an OPO condensate requires a laser energy slightly blueshifted with respect to the LP branch [17]. Above the OPO threshold pump-pump polariton interactions shift the LP branch into resonance with the laser [17]. Under a SAW, this blueshift is larger at the minima of the SAW potential, thereby leading to a higher pump polariton density at these positions. This spatial modulation leads to diffraction replicas of the pump beam with $k_{y}= \pm k_{\mathrm{SAW}}$ and a measured intensity of $0.5 \%$ of $I_{p}$, from which we estimate a pump density modulation amplitude of about $20 \%$. The diffraction replicas may undergo a parametric process with the unperturbed pump state at $k_{y}=0$, namely, $\quad k_{y}^{(p)}(=0)+k_{y}^{(p)}\left(=k_{\mathrm{SAW}}\right) \rightarrow k_{y}^{(s)}\left(=k_{\mathrm{SAW}} / 2\right)+$ $k_{y}^{(i)}\left(=k_{\mathrm{SAW}} / 2\right)$ thus providing the observed parametric gain at $k_{y}= \pm k_{\mathrm{SAW}} / 2$. The coupling (and coherence) between adjacent wires reduces at higher SAW amplitudes $\left(u \sim 5.7 \mathrm{~mW}^{1 / 2}\right)$ due to the stronger confinement. The momentum of the condensate is thus no longer well defined and the emission pattern in momentum space spreads over the whole MBZ branch [Fig. 2(c)].

The condensate wires were directly detected using a correlation setup to measure the classical second order intensity autocorrelation function $g^{(2)}(\delta y, \delta t)=$ $\left\langle I_{\mathrm{PL}}(0,0), I_{\mathrm{PL}}(\delta y, \delta t)\right\rangle$ of the PL intensity $\left(I_{\mathrm{PL}}\right)$ from two small spots $\left(2 \times 2 \mu \mathrm{m}^{2}\right)$ separated by a distance $\delta y$ [Fig. 2(d)]. As expected, the $g^{(2)}$ function exhibits maxima at time intervals $\delta t$ multiples of the SAW period $\tau_{\mathrm{SAW}}$, when condensates cross the collection regions of both detectors. Figure 2(e) displays $g^{(2)}$ functions recorded for different $\delta y$. The phase of the oscillations changes by $\pi$ when the spots are displaced by $\delta y=\lambda_{\mathrm{SAW}} / 2$ : the fact that the rate of change $\delta y / \delta \tau=v_{\text {SAW }}$ proves that the confined condensate regions are spatially separated by $\lambda_{\mathrm{SAW}}$ and move at $v_{\mathrm{SAW}}$. Quantitative information about the modulation amplitude can be obtained by assuming that $I_{\mathrm{PL}}(\delta y, \delta t)=I_{0}+\Delta I \sin \left(k_{\mathrm{SAW}} \delta y+\omega_{\mathrm{SAW}} \delta t\right), \quad$ yielding the $\quad g^{(2)}(\delta y, \delta t)=I_{0}^{2}+\Delta I^{2} / 2 \sin \left(k_{\mathrm{SAW}} \delta y+\omega_{\mathrm{SAW}} \delta t\right)$. From the maxima to minima ratio of about 1.25 in Fig. 2(e), we determine a signal population modulation $\left(\propto \Delta I / I_{0}\right)$ of $\sim 45 \%$. While these results demonstrate the confinement and motion of the condensate wires, they do not imply the long-range transport of a coherent state, since the measured coherence time of unperturbed condensates of 200-500 ps $[5,11]$ is significantly shorter than $\tau_{\mathrm{SAW}}=$ $2.65 \mathrm{~ns}$.

The coherence among the wire condensates was further investigated by recording first-order spatial correlation functions $g^{(1)}(\mathbf{r},-\mathbf{r})$ for the wire arrays using the procedure described in Ref. [1]. The full width at $1 / 2.7$ of the maxima of $g^{(1)}(\mathbf{r},-\mathbf{r})$ gives the corresponding coherence lengths $L_{x}$ and $L_{y}$ along the $x$ and $y$ directions, respectively. In the absence of a SAW, the emission of the condensate occurs at $k=0$ and is spatially homogeneous with equal $L_{y} \approx L_{x} \approx 25 \mu \mathrm{m}$ [cf. Figure 3(a)] limited by the size of the excitation spot. The spreading of the dispersion in $k$ space in Figs. 2(b) and 2(c) is accompanied by a reduction of both $L_{y}$ and $L_{x}$ [Fig. 3(b)].

The SAW modulation acting directly on the signal reduces the tunneling coupling between adjacent condensates, thus allowing their phases to evolve independently with the consequent collapse of $L_{y}$ to values close to $\lambda_{\mathrm{SAW}} / 2=4 \mu \mathrm{m}$ [18]. Using the measured polariton mass $m_{\mathrm{pol}}=4.5 \times 10^{-5} m_{e}$ ( $m_{e}$ is the free electron mass) and assuming a potential barrier $V_{\mathrm{SAW}}=0.16 \mathrm{meV}$ [cf. Fig. 1(e)] we estimate a tunneling time $\tau_{t}=h / J \sim 200 \mathrm{ps}$ $\left[J=4 V_{\mathrm{SAW}} \exp \left(-\sqrt{\left\{2 m_{\mathrm{pol}} V_{\mathrm{SAW}}\right\} \lambda_{\mathrm{SAW}}} / \hbar\right)\right.$ is the tunneling energy]. This time is comparable to the condensate coherence time, hence preventing the full establishment of spatial coherence between adjacent condensates.

The $20 \%$ spatial modulation of the pump (and hence modulation of the gain [19]) deduced from the diffraction replicas is also likely to contribute to the signal spatial modulation and hence to the reduction of its coherence. A $20 \%$ modulation is also expected for the signal density, given the measured linear dependence of the signal intensity on the pump power above threshold. We note, however, that we have observed a similar reduction of
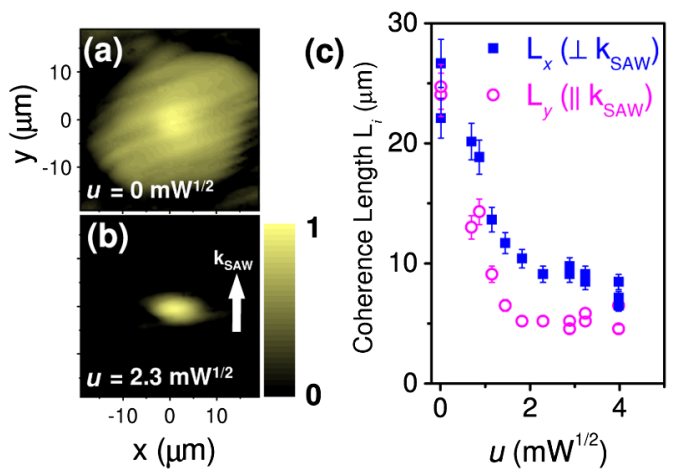

FIG. 3 (color online). First-order spatial correlation functions $g^{(1)}$ of condensates for (a) $u=0$ and (b) $u=2.3 \mathrm{~mW}^{1 / 2}$. (c) Dependence on SAW amplitude $u$ of the spatial coherence lengths $L_{x}$ and $L_{y}$ along the $x$ and $y$ directions, respectively. 


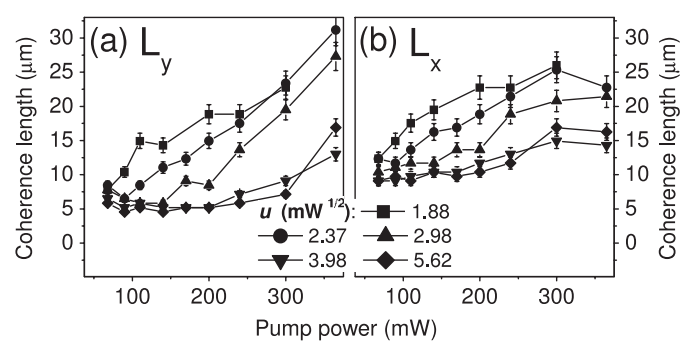

FIG. 4. Dependence of (a) $L_{y}$ and (b) $L_{x}$ on the pump power $I_{p}$ for different acoustic modulation amplitudes $u$.

coherence length with SAW power for condensates excited resonantly with the excitonic LP branch at a large incidence angle of $30^{\circ}$ [8]. In this case the direct modulation of the condensate by the SAW potential plays the major role in the reduction of its coherence, because the modulation of the pump states by the SAW is negligible due to the broad exciton resonance (FWHM $\sim 1 \mathrm{meV}$ ).

The spatial coherence in Figs. 3(b) and 3(c) also reduces along the wires ( $x$ direction). We suggest that this is associated with phase fluctuations in the 1D polariton system. This phenomenon is well known at thermodynamic equilibrium $[2,20]$ and has also been predicted for nonequilibrium systems such as the 1D OPO [21]. For the extended OPO the phase fluctuations are negligible and the signal coherence is predicted to be limited only by the size of the excitation spot [22]. For a nonequilibrium condensate the phase fluctuations are predicted to determine the coherence length, which becomes proportional to $1 / \Gamma$, where $\Gamma$ is the decay rate of excitations (diffusive Goldstone mode) in the condensate [21]. $\Gamma$ is estimated to be of the order of the inverse polariton lifetime and plays a role analogous to the temperature in equilibrium systems. We note that coherence lengths of the order of $100 \mu \mathrm{m}$ have recently been reported in nonresonantly pumped $1 \mathrm{D}$ condensates in high- $Q$ microresonators $(Q \sim 15000)$ [23]. The lower $L_{x}$ values in our wires can be attributed to the lower $Q$ (and, consequently, the shorter lifetimes and higher $\Gamma$ ) in our microcavities. Also, the small excitation spot in Ref. [23] ( $\sim 3 \mu \mathrm{m})$ is expected to reduce noise due to spatial decoupling of propagating polaritons from the pump reservoir, thus potentially increasing the coherence length.

We show finally that the degree of lateral confinement of the wires can be reversed by increasing the polariton density. As presented in Fig. 4, for a fixed SAW amplitude both coherence lengths $L_{y}$ and $L_{x}$ increase with $I_{p}$. Repulsive (signal-signal and signal-pump) interactions, which are stronger in the minima than in the maxima of the SAW potential, raise the energy of the trapped condensates above the percolation limit. As a result, the confinement effects induced by the SAW are partially reversed and the long-range spatial coherence of the signal is reestablished, as observed in Fig. 4. In agreement with the screening mechanism, the effects are more pronounced for low acoustic powers, where the energy renormalization due to polariton-polariton interactions more easily overcomes the energies barriers imposed by the SAW potential.

In conclusion, we have demonstrated that the coherent spatial modulation of polariton condensates by a SAW creates a condensate fragmented in the form of wires aligned along the wave fronts. These results open the way for the dynamic manipulation of polaritonic quantum phases by tunable periodic potentials necessary for computational schemes [24] and for the investigation of manybody phenomena such as Josephson coupling and, for shorter SAW wavelengths, condensate-Mott insulator transition [7]. Finally, the acoustic transport of coherent polariton states can be realized in high $Q$-microcavities with longer condensate coherence times.

We would like to thank F. Große for discussions as well as M. Höricke, W. Seidel, and E. Wiebecke for the fabrication of the samples. E. A. C.-M. would like to thank the Deutscher Akademischer Austausch Dienst for partial financial support.

[1] J. Kasprzak et al., Nature (London) 443, 409 (2006); R. Balili et al., Science 316, 1007 (2007)

[2] L. Pitaevskii and S. Stringari, Bose-Einstein Condensation (Clarendon, Oxford, 2003).

[3] M. Wouters and I. Carusotto, Phys. Rev. Lett. 99, 140402 (2007).

[4] K. G. Lagoudakis et al., Nature Phys. 4, 706 (2008).

[5] A. P. D. Love et al., Phys. Rev. Lett. 101, 067404 (2008).

[6] A. Amo et al., Nature (London) 457, 291 (2009).

[7] M. Greiner et al., Nature (London) 415, 39 (2002); F. S. Cataliotti et al., Science 293, 843 (2001)

[8] C. W. Lai et al., Nature (London) 450, 529 (2007).

[9] M. Remeika et al., Phys. Rev. Lett. 102, 186803 (2009).

[10] R. M. Stevenson et al., Phys. Rev. Lett. 85, 3680 (2000).

[11] D. N. Krizhanovskii et al., Phys. Rev. Lett. 97, 097402 (2006).

[12] M. Wouters and I. Carusotto, Phys. Rev. A 76, 043807 (2007).

[13] J. Rudolph, R. Hey, and P. V. Santos, Phys. Rev. Lett. 99, 047602 (2007).

[14] M. M. de Lima et al., Phys. Rev. Lett. 97, 045501 (2006).

[15] M. M. de Lima, Jr. and P. V. Santos, Rep. Prog. Phys. 68, 1639 (2005).

[16] D. N. Krizhanovskii et al., Phys. Rev. B 77, 115336 (2008).

[17] D. M. Whittaker, Phys. Rev. B 71, 115301 (2005).

[18] The spatial resolution of our setup is $(\sim 5 \mu \mathrm{m}) ; L_{y}$ may actually be smaller.

[19] G. Roumpos et al., Phys. Rev. Lett. 104, 126403 (2010).

[20] S. Dettmer et al., Phys. Rev. Lett. 87, 160406 (2001).

[21] M. Wouters and I. Carusotto, Phys. Rev. B 74, 245316 (2006).

[22] I. Carusotto and C. Ciuti, Phys. Rev. B 72, 125335 (2005).

[23] E. Wertz, et al., arXiv:1004.4084.

[24] D. Jaksch, et al., Phys. Rev. Lett. 82, 1975 (1999). 